\title{
Design of an instrument for measuring the spectral bidirectional scatter distribution function
}

\author{
Frédéric B. Leloup, ${ }^{1, *}$ Stefaan Forment, ${ }^{1}$ Philip Dutré, ${ }^{2}$ \\ Michael R. Pointer, ${ }^{3}$ and Peter Hanselaer ${ }^{1}$ \\ 1 Light and Lighting Laboratory, KaHo Sint-Lieven, Gebroeders Desmetstraat 1, B-9000 Gent, Belgium \\ ${ }^{2}$ Computer Graphics Group, Department of Computer Science, K.U. Leuven, Celestijnenlaan 200A, B-3001 Leuven, Belgium \\ ${ }^{3}$ Department of Colour Science, Faculty of Mathematics and Physical Sciences, University of Leeds, Leeds LS2 9JT, UK \\ ${ }^{*}$ Corresponding author: frederic.leloup@kahosl.be
}

Received 16 May 2008; accepted 17 August 2008;

posted 29 August 2008 (Doc. ID 96326); published 8 October 2008

\begin{abstract}
The spectral bidirectional scatter distribution function (BSDF) offers a complete description of the spectral and spatial optical characteristics of a material. Any gloss and color measurement can be related to a particular value of the BSDF, while accurate luminaire design with ray tracing software requires the BSDF of reflectors and filters. Many measuring instruments, each having particular advantages and limitations, have been reported in the literature, and an overview of these instruments is included. A measuring instrument that allows for an absolute determination of the spectral BSDF with a full three dimensional spatial coverage in both reflectance and transmittance mode, a broadband spectral coverage, a large dynamic range, a reasonable acquisition time, and a large sample illumination area is presented. The main instrument characteristics are discussed, and the measurement capabilities are illustrated. (C) 2008 Optical Society of America
\end{abstract}

OCIS codes: $\quad 120.5820,290.1483,290.5845$.

\section{Introduction}

The spectral bidirectional scatter distribution function (BSDF) characterizes the scattering properties of a material for any angle of illumination or viewing and offers a complete description of the spectral and spatial optical characteristics. An accurate determination of the BSDF function is important for many scientific applications and technological developments.

The measurement and characterization of surface roughness was one of the first important applications of BSDF. A correlation was demonstrated between the BSDF and the power spectral density function (PSD) from surface profile data on optically smooth surfaces [1].

With the strong progress made in computer science, other applications became important: photo-

0003-6935/08/295454-14\$15.00/0

(C) 2008 Optical Society of America realistic rendering (computer graphics) [2-12], the characterization of ground surfaces for remote sensing [13-17], daylight simulation in buildings with advanced fenestration materials $[18,19]$, road materials [20], paper [21-25], polarimetry [26], and architectural and lighting design [27-30].

Luminaire design using ray tracing has become an important development tool in the lighting industry. Simulation programs require geometrical, spatial, and spectral input characteristics of the light source and of the optical materials such as reflectors, filters, and diffusors. The accuracy of the simulations depends highly on the availability of the BSDF of these materials.

As the BSDF offers basic input for the visual appearance properties of materials, any color or gloss measurement can be related to a particular value of the general spectral BSDF. While color is related to the spectral properties of the light both absorbed and scattered within a medium, gloss is related to 
the directional dependency of the light reflected from the surface. With respect to standardization of measurements, the Commission Internationale de l'Eclairage (CIE) and American Society for Testing and Materials (ASTM) have recommended a number of basic geometries defining the illumination and viewing angles [31-35]. These specific geometries are usually implemented in commercially available color and gloss measuring instruments. There are, however, many materials that cannot be adequately measured using such relatively limited conditions. For example, gonio-apparent or special-effect surface colors, which change in color according to the angle of illumination and viewing, have rapidly grown in popularity over the past thirty years, and nowadays dramatic color effects can be achieved on automobiles and sportsware, among others [36].

Many BSDF measuring instruments, each having particular advantages and limitations, have been reported in the literature, and an overview of these instruments is included. In this paper, a measuring instrument allowing for an absolute determination of the spectral BSDF, with a full three dimensional (3D) spatial coverage in both reflectance and transmittance mode, a broadband spectral coverage, a large dynamic range, a reasonable acquisition time, and a large sample illumination area is presented. Finally, some test sample measurements are presented and discussed, illustrating the capabilities of the instrument.

\section{Instrument Overview}

The bidirectional reflectance distribution function (BRDF) and the bidirectional transmittance distribution function (BTDF) characterize the scattering properties of a material in reflection and transmission modes, respectively. Both functions are included in the BSDF. The introduction of the concept and notation of the BRDF is accredited to Nicodemus et al. [37]. BRDF was defined in radiometric terms as the surface radiance of a sample, in a particular viewing direction, due to the scattering of the radiation incident from a particular direction of irradiation:

$$
q_{e, \lambda}\left(\theta_{i}, \phi_{i}, \theta_{s}, \phi_{s}, \lambda\right)=\frac{\mathrm{d} L_{e, \lambda, s}\left(\theta_{i}, \phi_{i}, \theta_{s}, \phi_{s}, \lambda\right)}{\mathrm{d} E_{e, \lambda, i}\left(\theta_{i}, \phi_{i}, \lambda\right)}\left[\mathrm{sr}^{-1}\right],
$$

with $q_{e, \lambda}$ the spectral BRDF, $\left(\theta_{i}, \phi_{i}\right)$ the spherical coordinates of the light incident on the surface, $\left(\theta_{s}, \phi_{s}\right)$ the spherical coordinates of the light scattered from the surface, $\mathrm{d} L_{e, \lambda . s}$ the differential spectral radiance, and $\mathrm{d} E_{e, \lambda, i}$ the differential spectral irradiance from a differential solid angle. The spherical coordinates are referenced to the surface normal.

Serious efforts have been made to develop accurate and efficient bidirectional goniometric measurement devices. Almost all measurement devices described in the literature are based on a scanning process, i.e., the sample, detector, or source perform relative individual movements to cover all incoming and outgoing light flux directions for which BSDF data are needed. The ability to achieve this broad angular coverage is realized by means of a goniometer. While most measurement devices are restricted to measurements in directions within the plane of incidence, or so-called uniplanar devices, during the past decade instruments able to perform out-of-plane measurements have been reported. White et al. [38] and Germer (the Goniometric Optical Scatter Instrument, GOSI) [39] have built a device with excellent angular coverage, but the limited spectral coverage (only three wavelengths) and long measurement time (of the order of several hours) constitute important restrictions.

Broadband spectral coverage can be achieved by the use of spectral filtering at the source (monochromatic illumination) or at the detector (polychromatic illumination). Monochromatic illumination was applied by Obein et al. [40], who used a light source with a dual grating monochromator to provide radiation from $360 \mathrm{~nm}$ to $780 \mathrm{~nm}$ and a spectral bandwidth of $0.5 \mathrm{~nm}$. With polychromatic illumination, the detection unit consists of a scanning monochromator/ detector combination $[2,41]$, or a spectrograph that measures the entire spectrum instantaneously $[\underline{4}, 5,10,11,42,43]$.

To reduce acquisition time, multiple angles can be detected simultaneously. This can be achieved by use of a camera combined with optical accessories, as demonstrated by Ward [2] and Dana et al. [6]. Ward developed an image gonioreflectometer that samples two dimensions of the BRDF simultaneously by means of a mirrored hemisphere used as a projection surface and a fish-eye lens for the image capture. Ward's instrument, although limited by its optics and by vignetting issues at near-grazing angles, has a measurement time reduced to the order of minutes. Dana et al. used a robotic manipulator and a CCD camera moved to a maximum of seven different locations, representing 205 different combinations of viewing and illumination directions. A BRDF measurement database with reflectance measurements from 61 different samples was reported [44]. Many others used a similar concept $[3,9,12,19,2 \overline{8,45}]$.

The use of a camera to spee $\bar{d}$ acquisition was further developed by Lu et al. [46], measuring velvet samples mounted on a cylinder. The use of a known curved geometry improves the efficiency of data acquisition because a stream of data points can be acquired from one scanned image. Marschner et al. [7] reported a similar device with a spherical material sample, thus allowing for measurements over the complete hemisphere. Recently, several other instruments have been reported based on this concept $[24,47,48]$.

Most of the instruments cited are designed to measure in reflectance mode; only a few instruments (Andersen [19], Goldstein [26], and Obein et al. [40]) allow measurement in transmittance mode. Moreover, each instrument is developed with a specific application in mind. As a consequence, the design is 
usually optimized for a specific parameter, restricting the other device characteristics.

\section{Instrument Design}

\section{A. Requirements}

Our aim was to develop a BSDF measurement instrument with the following basic requirements:

- A large sample illumination area

- Absolute determination of the spectral BSDF

- A reasonable acquisition time

- Full 3D spatial coverage in both reflectance and transmittance mode.

The diameter of the illumination area on the sample is determined by the spatial uniformity of the sample to be measured. Initially, the instrument was developed to measure the mean scattering of macrostructured surfaces, for example, the hammer-scale reflectors of luminaires, with a spatial periodicity of several $\mathrm{mm}$. In order to obtain a reasonable average value for the surface, an illumination area with a diameter of at least two centimeters was required.

In order to perform accurate absolute measurements of specularly as well as diffusely scattering samples, the incident and scattered light has to be measured preferentially with the same detector head [41]. Consequently, when measuring the incident flux, the detector head aperture must be at least slightly larger than the diameter of the light beam. The front surface of the detector head is composed of a fused silica plano-convex lens with an iris diaphragm mounted immediately in front of it. The aperture of this diaphragm is larger than the diameter of the light beam. This rather wide detector aperture compromises the angular resolution of the instrument. Limiting the angular aperture from the detector as seen from the sample to a maximum of $2^{\circ}$, the distance between the sample and detector can be calculated to be at least $0.7 \mathrm{~m}$.

The requirement for absolute measurements imposes another important condition on the measurement setup with respect to the dynamic range and the signal-to-noise ratio. The largest signal will be recorded when making a measurement of the incident spectral flux from the light source $\Phi_{e, \lambda, i}$. The minimum response will be recorded when making measurements of diffuse black samples. For characterizing diffuse black materials with a reflectance of $5 \%$, a dynamic range exceeding six decades is required (see Appendix A). The dynamic range of a CCD detector is considerably smaller, and the use of neutral density filters with optical densities up to a value of three, in combination with an optimized integration time, will be necessary.

The acquisition time is another important issue for BSDF measurements. The need for a reasonable acquisition time excludes a combination of a spectral as well as a spatial scanning instrument. Since absolute measurements imply a spatial scanning technique, a spectrometer with a CCD detector is preferred above a monochromator/photomultiplier combination for signal detection. An ozone-free xenon light source was installed to provide a high intensity broadband irradiation. Because of the large emission intensities in the blue-violet region of the visual spectrum, a much improved signal-to-noise ratio can be achieved with xenon illumination compared to tungsten-halogen illumination. Correction for both short- and longterm drift will, however, be required.

The weight of a spectrometer/CCD recording unit restricts its location in the goniometer; to prevent bending of the detection arm, the spectrometer was positioned at the center of rotation of the detector bench. A high-grade fused silica fiber bundle was used to guide the radiation collected by the detector lens to the spectrometer. The rectangular fiber end was used to replace the input slit of the spectrometer. If the circular end of the fiber bundle is used as a field stop for the detector lens, this would result in a very nonuniform entrance window. For this reason, a small integrating cylinder was introduced between the detector lens and the fiber bundle, which unfortunately decreases the level of response of the detection system.

For the characterization of anisotropic samples at oblique angles of incidence, measurements restricted to the plane of incidence offer incomplete information. Typical examples are samples of ruled (or brushed) aluminum. Ray tracing software for luminaire design makes use of these anisotropic BRDF characteristics of aluminum reflectors or baffles. A full 3D instrument with two degrees of freedom for the detection arm can provide complete datasets.

\section{B. General Description}

\section{Introduction}

According to ASTM E1392 [49], the practical formula used to determine the absolute BSDF under the condition that the field-of-view of the receiver field stop is sufficiently large to include the entire illuminated area for all angles of interest can be written as

$$
q_{e, \lambda}=\frac{\Phi_{e, \lambda, s}}{\Phi_{e, \lambda, i} \Omega_{s}\left|\cos \theta_{s}\right|}
$$

with $\Phi_{e, \lambda, i}$ and $\Phi_{e, \lambda, s}$ the spectral density of the radiant flux incident on the sample and received by the detector, respectively, and $\Omega_{s}$ being the solid angle subtended by the receiver aperture stop from the sample origin. According to Eq. (2), the illuminated area on the sample need not be known. In fact, the ASTM method does not define the BRDF as a spectral quantity. With the introduction of spectral densities of radiant flux, the wavelength dependency is included in Eq. (2). Because the incident and scattered flux is measured consecutively with the same 
detector head, the flux ratio is equal to the ratio of the detector responses at each wavelength.

Equation (2) is valid for diffusely scattering samples. For specular samples, the detector aperture is underfilled, and the reflectance or transmittance can be calculated as

$$
\frac{\Phi_{e, \lambda, s}}{\Phi_{e, \lambda, i}}
$$

The overall optical layout of the designed instrument is presented in Fig. 1. The system consists of two major sections: the illumination section and the detection section.

\section{Illumination Section}

The illumination section includes a fixed $250 \mathrm{~W}$ xenon lamp (Osram XBO 250 W OFR 18.0 A VS1), mounted in a lamp house (Müller GmbH LAX 1450) and connected to a power supply (Müller $\mathrm{GmbH}$ SVX1450). The dimensions of the discharge are $0.7 \mathrm{~mm} \times 1.7 \mathrm{~mm}$ (width $\times$ height). The lamp can be adjusted with two micrometer screws at the back of the lamp house. Additionally, a spherical reflector (spherical rear mirror RS, $6 \mathrm{~mm}$ focus) images the arc on to the lamp, enabling an increase in the light-intensity output through the condenser lens of approximately $30 \%$. Two supplementary micrometer screws enable the exact positioning of the reflected image between the lamp electrodes.

The power supply provides a constant current to the lamp of $18 \mathrm{~A}$ with a ripple of $0.2 \%$ pp (typical at $20 \mathrm{~A}$, max $0.5 \%$ ). The current supplied can be regulated in the range 10 to $30 \mathrm{~A}$. The power supply stability is equal to $20 \mathrm{~mA}$ within any $8 \mathrm{~h}$ period.

The condenser lens (Müller LAX1450-11 Spectrosil B., focal length $50 \mathrm{~mm}$ ) images the discharge onto a concave mirror (Newport 20DC1000AL.2, $500 \mathrm{~mm}$

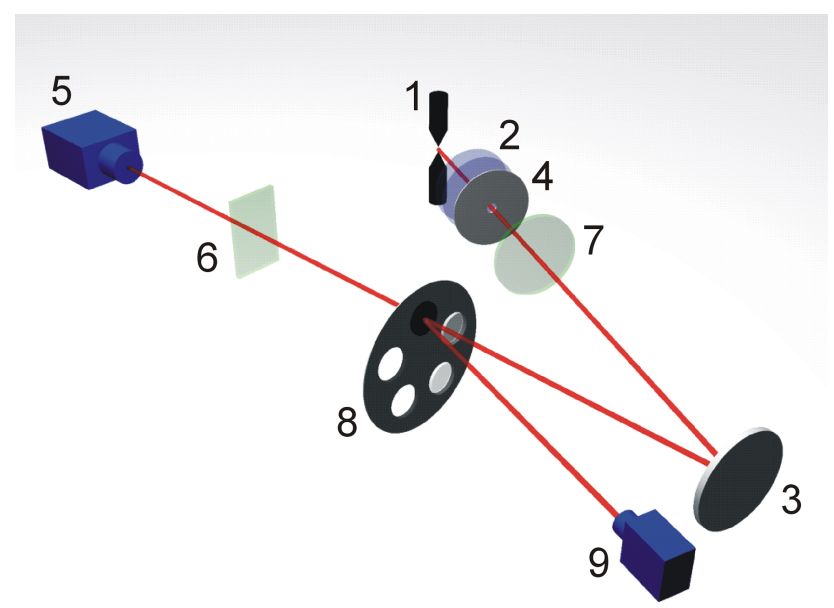

Fig. 1. (Color online) Optical layout of the instrument: (1) xenon discharge source, (2) condenser lens, (3) concave mirror, (4) iris diaphragm, (5) detector head, (6) sample holder, (7) long-wave pass filter, (8) filter wheel, and (9) reference detector. focal length), placed at a distance of $0.7 \mathrm{~m}$. This avoids the reimaging of the nonuniform radiance of the discharge. An adjustable diaphragm (Müller MIB50) is positioned behind the condenser lens. The diaphragm selects the central part of the condenser lens and acts as a secondary light source with a more uniform radiance distribution. This diaphragm is imaged by the concave mirror onto the front aperture of the detector when the latter is aligned with the incident beam.

A concave mirror is preferred to a lens to avoid chromatic aberration and to limit the dimensions of the instrument. In order to minimize the introduction of off-axis aberrations, the tilt of the mirror is kept to a minimum.

Limiting the angular aperture of the detector as seen from the sample to a maximum of $2^{\circ}$, the distance between the sample plane and detector must be at least $0.7 \mathrm{~m}$ as described above. In order to measure in reflectance mode as well as in transmittance mode, the minimum distance between the mirror and the detector has to be $1.5 \mathrm{~m}$. This results in a mirror focal length of at least $425 \mathrm{~mm}$. A mirror with a focal length of $500 \mathrm{~mm}$ was installed. The corresponding image distance was $288 \mathrm{~cm}$, and the absolute magnification of the illumination design was $13 \times$. Taking into account the detector aperture of $1 \mathrm{in}$. $(1 \mathrm{in} .=2.54 \mathrm{~cm})$, the aperture of the diaphragm in front of the condenser lens must be less than $2 \mathrm{~mm}$.

Before reaching the sample, the incident beam passes through a long-wave pass filter (Schott CGGG-385) to eliminate second-order wavelengths over the visible spectrum and to absorb the UV radiation from the light source.

Additionally, the light beam passes through an automated filter wheel with five possible filter positions: blank, opaque, and three neutral density (ND) filters. A readout of the "dark" signal is performed when the opaque filter obstructs the beam. The reflectance of this opaque filter also provides a reference beam that is measured by a silicon photodiode. In this way, short-term fluctuations and long-term drift of the xenon lamp output can be monitored and corrected.

Polarized incident irradiation can complicate the measurements at oblique angles of incidence. The degree of polarization was checked with a rotating polarizer and found to be negligible. If necessary, the setup allows for the insertion of a polarizer or depolarizer in the optical path of the incident or scattered beam.

The uniformity of the illumination spot at the sample position was checked using an absolute luminance and colorimetric camera (MURATest 2111 by Eldim). The luminance distribution on a white Lambertian sample is shown in Fig. 2. The lines represent isoluminance regions (expressed in $\mathrm{cd} / \mathrm{m}^{2}$ ). A representation of the luminance values of each pixel on a horizontal cut in the center of the image is presented in the graphs on the right of Fig. 2 (on a linear and a log scale, respectively). Over the whole 

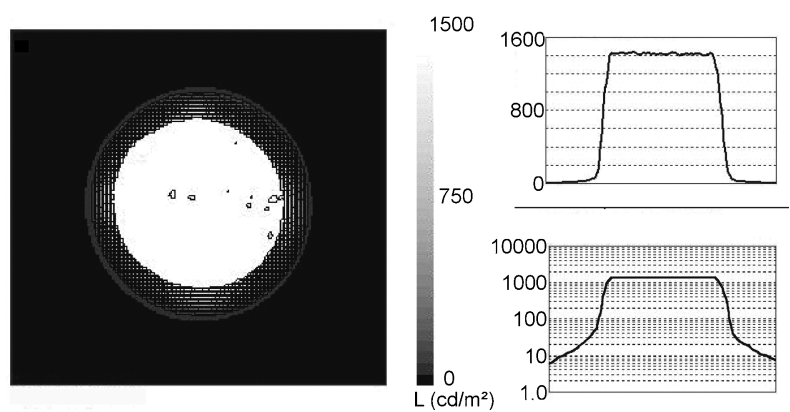

Fig. 2. Uniformity of the illumination spot at the sample position, determined by recording the luminance of a white Lambertian sample. Isoluminance regions (expressed in $\mathrm{cd} / \mathrm{m}^{2}$ ) are represented. On the right, luminance values of each pixel on a horizontal cut in the center of the image are displayed on a linear and a log scale (upper and lower figures, respectively).

illumination spot, luminance variations within $10 \%$ were achieved.

\section{Detection Section}

The detection section includes a detector head, located at $0.8 \mathrm{~m}$ from the sample plane. It is composed of a $40 \mathrm{~mm}$ fused silica plano-convex lens (focal length $63 \mathrm{~mm}$ ), coated with an antireflective layer to prevent back reflection onto the sample, and an iris diaphragm (diameter 1 in.) placed immediately in front. The diameter of the image of the secondary light source is smaller than the maximum aperture of the detector head. In this way, the complete incident or specularly reflected or transmitted flux can be measured with only one position of the detector. The spectral reflectance and transmittance of specular samples can easily be obtained using Eq. (3).

The detector lens images the sample area onto an aperture (diameter $2 \mathrm{~mm}$ ) drilled in the wall of a small cylindrical integrating cavity with internal diameter $5 \mathrm{~mm}$. This aperture acts as a field stop and determines the entrance window, which is larger than the illuminated area on the sample. The solid angle subtended by the receiver aperture stop from the sample origin is $7.5 \times 10^{-4} \mathrm{sr}$, corresponding to a plane acceptance angle of $1.8^{\circ}$. This angular resolution can be improved by increasing the distance or by decreasing the detector diaphragm aperture, losing the possibility of absolute measurements.

In the base of the cavity, a circular high-grade fused silica fiber bundle is positioned. The incoming radiation is outside the acceptance cone of the fiber. The other, rectangular, fiber end termination $(200 \mu \mathrm{m}$ by $6 \mathrm{~mm}$ ) is coupled to the $1 / 8 \mathrm{~m}$ focal length Oriel spectrometer. The (interchangeable) grating has a line density of 400 lines $/ \mathrm{mm}$. At the spectrometer exit plane, a 1 in. Andor open electrode, cooled $\left(-20{ }^{\circ} \mathrm{C}\right) \mathrm{CCD}$ detector is mounted. With a $1024 \times$ 256 pixel array and full vertical binning, the spectral resolution is $4 \mathrm{~nm}$, and the complete visible spectrum is recorded. Data acquisition with full vertical binning, dark current correction and an integration time set for optimum signal-to-noise ratio is controlled by software.

The spectrograph/CCD combination is mounted on the rotation axis of the detector bench. In this way, the inertia can be kept low and the fiber curvature remains unchanged during measurements.

\section{Angular Setup}

Figure 3 represents the sample holder geometry. The sample is mounted on rotation stage $\mathrm{D}$ (angle $\delta$ ) with a horizontal rotation axis. The sample reference surface can be adjusted to coincide with the center of the goniometer using translation stage $\mathrm{T}$, thus allowing for variable sample thickness. The complete sample holder is mounted on rotation stage $\mathrm{C}$ (angle $\gamma$ ).

Stage $C$ has a resolution of $1^{\prime}$, while the resolution of stage $\mathrm{D}$ is equal to $1^{\circ}$. The rotation axis of $\mathrm{C}$ is always fixed in space, while the rotation axis of $D$ is not. The translation table enables a translation travel of $25 \mathrm{~mm}$, with a resolution of $0.01 \mathrm{~mm}$. The sample holder has a clear aperture allowing the incident beam to pass through when measuring the incident flux and the BTDF.

The detector unit is mounted at one end of an optical bench with length $2 \mathrm{~m}$ (Fig. 4). The middle of the bench is fixed to rotation stage $\overline{\mathrm{B}}$ (angle $\beta$ ), which in turn is mounted on a large bracket. The bracket is positioned on the platform of rotation stage A (angle $\alpha$ ). The rotation axis of $\mathrm{A}$ is always fixed in space, while the axis of $\mathrm{B}$ is not. The clear aperture of $\mathrm{A}$ allows an independent positioning of both the detector and the sample. In this way, any viewing angle can be established. Both motorized rotation stages

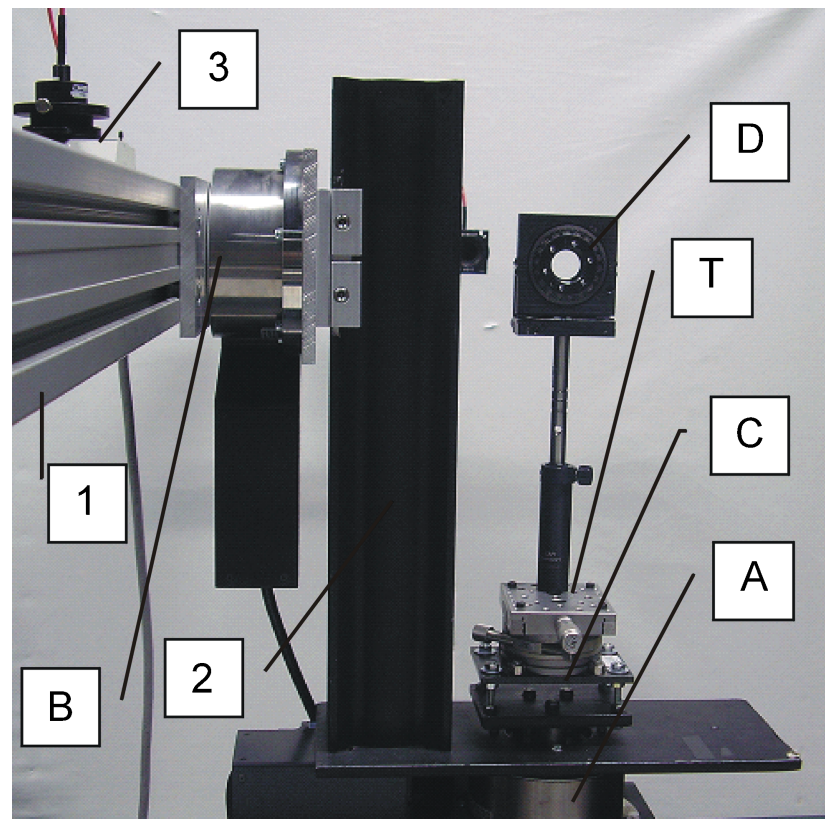

Fig. 3. (Color online) Sample holder with two rotation stages $\mathrm{C}$ and $\mathrm{D}$ (angles $\gamma$ and $\delta$ ) and a translation stage T. The detector bench (1) is mounted on rotation stage $\mathrm{B}$ (angle $\beta$ ); this unit is fixed with a bracket (2) onto rotation stage $\mathrm{A}$ (angle $\alpha$ ). The spectrometer with a CCD detector (3) is mounted onto the detector bench. 


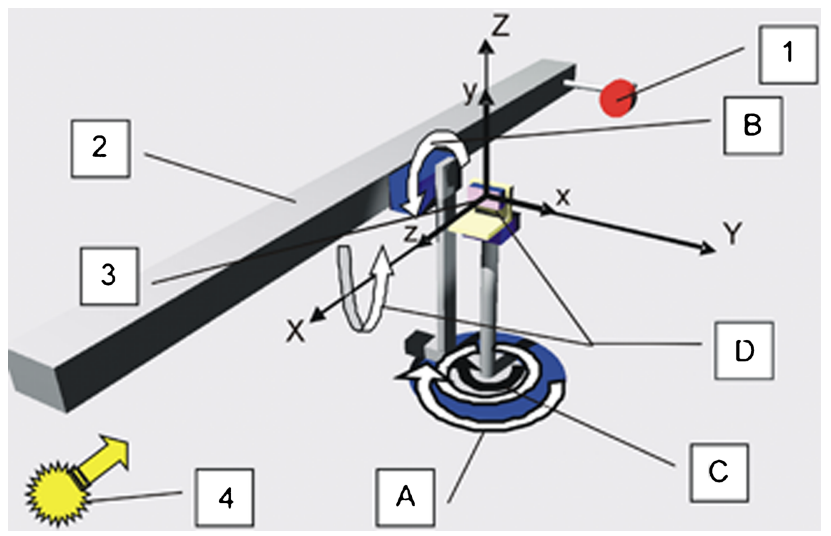

Fig. 4. (Color online) Representation of the initial (reference) position of the detector head (1), detector bench (2), and sample $x y z$ coordinate system (3) with respect to the global $X Y Z$ coordinate system. The incident light direction (4) and the positive sense of rotation of the four rotation stages $A, B, C$, and $D$ are also depicted.

(Newport RV 120PE) are equipped with an UE41PP stepper motor with an angular resolution of $0.001^{\circ}$. The angular range for which the fixed incident light beam will be obstructed by the detector head is restricted to less than $5^{\circ}$ horizontally and $3.6^{\circ}$ vertically.

The alignment of the four rotation stages, the sample holder, and the illumination optics is performed using three orthogonal laser beams. The lasers are fixed to the ceiling and the walls of the laboratory. External and internal reflections on right-angle prisms are used to check their positions. The laser beams define a rectangular reference system with the origin at the center of the goniometer. The four rotation axes are aligned with this reference system.

\section{Coordinate Systems}

A fixed global coordinate system (GCS) $X Y Z$ is chosen with the origin at the center of the goniometer, as shown in Fig. $\underline{4}$. The $X$ axis points toward the fixed light source, and the $Z$ axis is vertical. A second system, the sample coordinate system (SCS) $x y z$, is assigned to the sample, with the $z$ axis perpendicular to the sample surface. The reference position of the sample coordinate system, the detector, and the incident beam with respect to the global $X Y Z$ system is shown in Fig. 4. In this subsection, the conversion formulas from the rotation stage angles $(\alpha, \beta, \gamma, \delta)$ to the spherical angles in the SCS $\left(\theta_{i}, \phi_{i}, \theta_{s}, \phi_{s}\right)$ are established.

The initial directions of the light source, detector, and SCS relative to the GCS are $\mathbf{r}_{l, \text { ini }}, \mathbf{r}_{d, \text { ini }}$ and $\mathbf{e}_{x, \text { ini }}$, $\mathbf{e}_{y, \text { ini }}, \mathbf{e}_{z, \text { ini }}$, respectively. Rotation of the detector using $A$ and $B$, and of the sample using $C$ and $D$, will change these initial positions into the actual positions $\mathbf{r}_{l}, \mathbf{r}_{d}$ and $\mathbf{e}_{x}, \mathbf{e}_{y}, \mathbf{e}_{z}$, respectively. These final directions relative to the GCS can be written as

$$
\begin{aligned}
& \mathbf{r}_{l}=\mathbf{r}_{l, \text { ini }}, \quad \mathbf{r}_{d}=\mathbf{R}(-\mathbf{Z}, \alpha) \cdot \mathbf{R}(\mathbf{Y}, \beta) \cdot \mathbf{r}_{d, \text { ini }} \\
& \mathbf{e}_{x}=\mathbf{R}(-\mathbf{Z}, \gamma) \cdot \mathbf{R}(\mathbf{X}, \delta) \cdot \mathbf{e}_{x, \text { ini }} \\
& \mathbf{e}_{x}=\mathbf{R}(-\mathbf{Z}, \gamma) \cdot \mathbf{R}(\mathbf{X}, \delta) \cdot \mathbf{e}_{y, \text { ini }} \\
& \mathbf{e}_{z}=\mathbf{R}(-\mathbf{Z}, \gamma) \cdot \mathbf{R}(\mathbf{X}, \delta) \cdot \mathbf{e}_{z, \text { ini }}
\end{aligned}
$$

The matrix $\mathbf{R}(\mathbf{A}, \theta)$ describes the linear transformation of a vector when rotating around vector $\mathbf{A}$ over a positive angle $\theta$. This matrix is calculated explicitly in Appendix B. The rotation axes used in Eq. (4) are chosen in accordance with the internal positive rotation directions of the rotation stages (Fig. 4).

In E q. (4), the rotation order can be reversed, but in that case, the second rotation axis will no longer coincide with an axis of the GCS system, which complicates the transformation matrix $\mathbf{R}$ but gives similar results.

The explicit expressions for the actual positions of the light source, detector, and SCS in the GCS are given by

$$
\begin{aligned}
& \mathbf{r}_{l}=\left(\begin{array}{l}
1 \\
0 \\
0
\end{array}\right), \mathbf{r}_{d}=\left(\begin{array}{ccc}
\cos \alpha & \sin \alpha & 0 \\
-\sin \alpha & \cos \alpha & 0 \\
0 & 0 & 1
\end{array}\right) \cdot\left(\begin{array}{ccc}
\cos \beta & 0 & \sin \beta \\
0 & 1 & 0 \\
-\sin \beta & 0 & \cos \beta
\end{array}\right) \cdot\left(\begin{array}{c}
-1 \\
0 \\
0
\end{array}\right)=\left(\begin{array}{c}
-\cos \alpha \cdot \cos \beta \\
\sin \alpha \cdot \cos \beta \\
\sin \beta
\end{array}\right), \\
& \mathbf{e}_{x}=\left(\begin{array}{ccc}
\cos \gamma & \sin \gamma & 0 \\
-\sin \gamma & \cos \gamma & 0 \\
0 & 0 & 1
\end{array}\right) \cdot\left(\begin{array}{ccc}
1 & 0 & 0 \\
0 & \cos \delta & -\sin \delta \\
0 & \sin \delta & \cos \delta
\end{array}\right) \cdot\left(\begin{array}{l}
0 \\
1 \\
0
\end{array}\right)=\left(\begin{array}{c}
\sin \gamma \cdot \cos \delta \\
\cos \gamma \cdot \cos \delta \\
\sin \delta
\end{array}\right), \\
& \mathbf{e}_{y}=\left(\begin{array}{ccc}
\cos \gamma & \sin \gamma & 0 \\
-\sin \gamma & \cos \gamma & 0 \\
0 & 0 & 1
\end{array}\right) \cdot\left(\begin{array}{ccc}
1 & 0 & 0 \\
0 & \cos \delta & -\sin \delta \\
0 & \sin \delta & \cos \delta
\end{array}\right) \cdot\left(\begin{array}{l}
0 \\
0 \\
1
\end{array}\right)=\left(\begin{array}{c}
-\sin \gamma \cdot \sin \delta \\
-\cos \gamma \cdot \sin \delta \\
\cos \delta
\end{array}\right) \\
& \mathbf{e}_{z}=\left(\begin{array}{ccc}
\cos \gamma & \sin \gamma & 0 \\
-\sin \gamma & \cos \gamma & 0 \\
0 & 0 & 1
\end{array}\right) \cdot\left(\begin{array}{ccc}
1 & 0 & 0 \\
0 & \cos \delta & -\sin \delta \\
0 & \sin \delta & \cos \delta
\end{array}\right) \cdot\left(\begin{array}{l}
1 \\
0 \\
0
\end{array}\right)=\left(\begin{array}{c}
\cos \gamma \\
-\sin \gamma \\
0
\end{array}\right) \text {. }
\end{aligned}
$$


Because incident angles $\theta_{i}, \phi_{i}$, and viewing angles $\theta_{s}, \phi_{s}$ are referenced to the SCS and not to the GCS, the positions of the light source $\mathbf{r}_{l}$ and the detector $\mathbf{r}_{d}$ have to be recalculated in the SCS. The columns of the transformation matrix $\mathbf{M}$ are made up of the coordinates of the new vectors $\mathbf{e}_{x}, \mathbf{e}_{y}$, and $\mathbf{e}_{z}$ :

$$
\mathbf{M}=\left(\begin{array}{ccc}
\sin \gamma \cdot \cos \delta & -\sin \gamma \cdot \sin \delta & \cos \gamma \\
\cos \gamma \cdot \cos \delta & -\cos \gamma \cdot \sin \delta & -\sin \gamma \\
\sin \delta & \cos \delta & 0
\end{array}\right)
$$

The coordinates of the directions of the incident beam and the detector in the SCS system $\mathbf{r}_{l}^{\prime}$, respectively, $\mathbf{r}_{d}^{\prime}$ are given by

$$
\begin{gathered}
\mathbf{r}_{l}^{\prime}=\mathbf{M}^{-1} \cdot \mathbf{r}_{l}=\left(\begin{array}{c}
\sin \gamma \cos \delta \\
-\sin \gamma \sin \delta \\
\cos \gamma
\end{array}\right), \quad \text { (7) } \begin{array}{r}
\text { of ten measurements. The CCD temp } \\
\text { the measurements was stabilized at }
\end{array} \\
\mathbf{r}_{d}^{\prime}=\mathbf{M}^{-1} \cdot \mathbf{r}_{d}=\left(\begin{array}{c}
-\cos \alpha \cdot \cos \beta \cdot \sin \gamma \cdot \cos \delta+\sin \alpha \cdot \cos \beta \cdot \cos \gamma \cdot \cos \delta+\sin \beta \cdot \sin \delta \\
\cos \alpha \cdot \cos \beta \cdot \sin \gamma \cdot \sin \delta-\sin \alpha \cdot \cos \beta \cdot \cos \gamma \cdot \sin \delta+\sin \beta \cdot \cos \delta \\
-\cos \alpha \cdot \cos \beta \cdot \cos \gamma-\sin \alpha \cdot \cos \beta \cdot \sin \gamma
\end{array}\right) .
\end{gathered}
$$

\section{Instrument Characteristics}

\section{A. Instrument Signature}

The instrument signature is determined by scanning the light beam with the detector head in the absence of any sample. From this measurement, the angular resolution, the dynamic range, and the stray light scattered from the instrument components can be derived. The instrument signature, corrected for dark current and fluctuations of the xenon light source, is shown in Fig. 5. In this figure, the responses at 400,550 , and $700 \mathrm{~nm}$ are plotted on a $\log$ scale from $-3^{\circ}$ to $3^{\circ}$ off-axis with respect to the incident beam. The detector head is rotated in the horizontal plane. Each measured value is an average of ten measurements. The CCD temperature during the measurements was stabilized at $-20^{\circ} \mathrm{C}$. A simi-
Finally, the spherical coordinates for the incident light direction and the direction of the detector head in the SCS, respectively $\left(\theta_{i}, \phi_{i}\right.$ and $\left.\theta_{s}, \phi_{s}\right)$, can be related to the Cartesian coordinates:

$$
\begin{aligned}
& \sin \theta_{i} \cos \phi_{i}=\sin \gamma \cos \delta, \\
& \sin \theta_{i} \sin \phi_{i}=-\sin \gamma \sin \delta, \quad \cos \theta_{i}=\cos \gamma
\end{aligned}
$$

$$
\begin{aligned}
& \sin \theta_{s} \cos \phi_{s}=-\cos \alpha \cos \beta \sin \gamma \cos \delta \\
& \quad+\sin \alpha \cos \beta \cos \gamma \cos \delta+\sin \beta \sin \delta, \\
& \quad \sin \theta_{s} \sin \phi_{s}=\cos \alpha \cos \beta \sin \gamma \sin \delta \\
& -\sin \alpha \cos \beta \cos \gamma \sin \delta+\sin \beta \cos \delta, \\
& \cos \theta_{s}=-\cos \alpha \cos \beta \cos \gamma-\sin \alpha \cos \beta \sin \gamma .
\end{aligned}
$$

By solving both sets of equations, two analytical solutions are found, for $\alpha$ and $\beta$ and for $\gamma$ and $\delta$. These two solutions express the excess of rotational freedom offered by two stages, both with a rotation range of $2 \pi$. In practice, both solutions are not exactly equivalent because of the presence of the large detector bench. As can be seen from Fig. 4, only one solution will be most advantageous with respect to preventing the incident light from reaching the sample. lar instrument signature is obtained with the detector head rotating in the vertical plane.

The instrument signature can be subdivided into three regions. The first region lies within a small angle interval of $\pm 0.2^{\circ}$ off-axis. In this interval, the incident beam is totally included into the aperture of the detector head. The neutral density filter with a transmittance of $0.1 \%$ is switched into the light beam, and an integration time of only $0.023 \mathrm{~s}$ is required to obtain a CCD output just below saturation (50 000 counts).

A detailed view of the measured response and the standard deviation is given in Fig. 6 . The standard deviation was calculated from 20 consecutive measurements of the incident flux at $0^{\circ}$ viewing angle and ranged from $2.2 \%$ (at $400 \mathrm{~nm}$ ), over $1.8 \%$ (at $550 \mathrm{~nm}$ ) to $1.6 \%$ (at $700 \mathrm{~nm}$ ). It can be seen from Fig. 6 that the variation of the measured values in the $\pm 0.2^{\circ}$ interval lies within these limits. The theoretical value of the standard deviation can be expressed as $N^{1 / 2}$, with $N$ the number of counts of the signal. With $N$ ranging from 30,000 to 50,000 counts over the visible wavelength range, and taking into account the measurement of the dark current, the combined theoretical standard deviation of a measurement ranges from 0.9 to $1.2 \%$. The error introduced by the uncertainty of the transmittance of the neutral density filter, determined in the same conditions with the same system, enhances the total uncertainty budget to fall within the range 1.8 to 


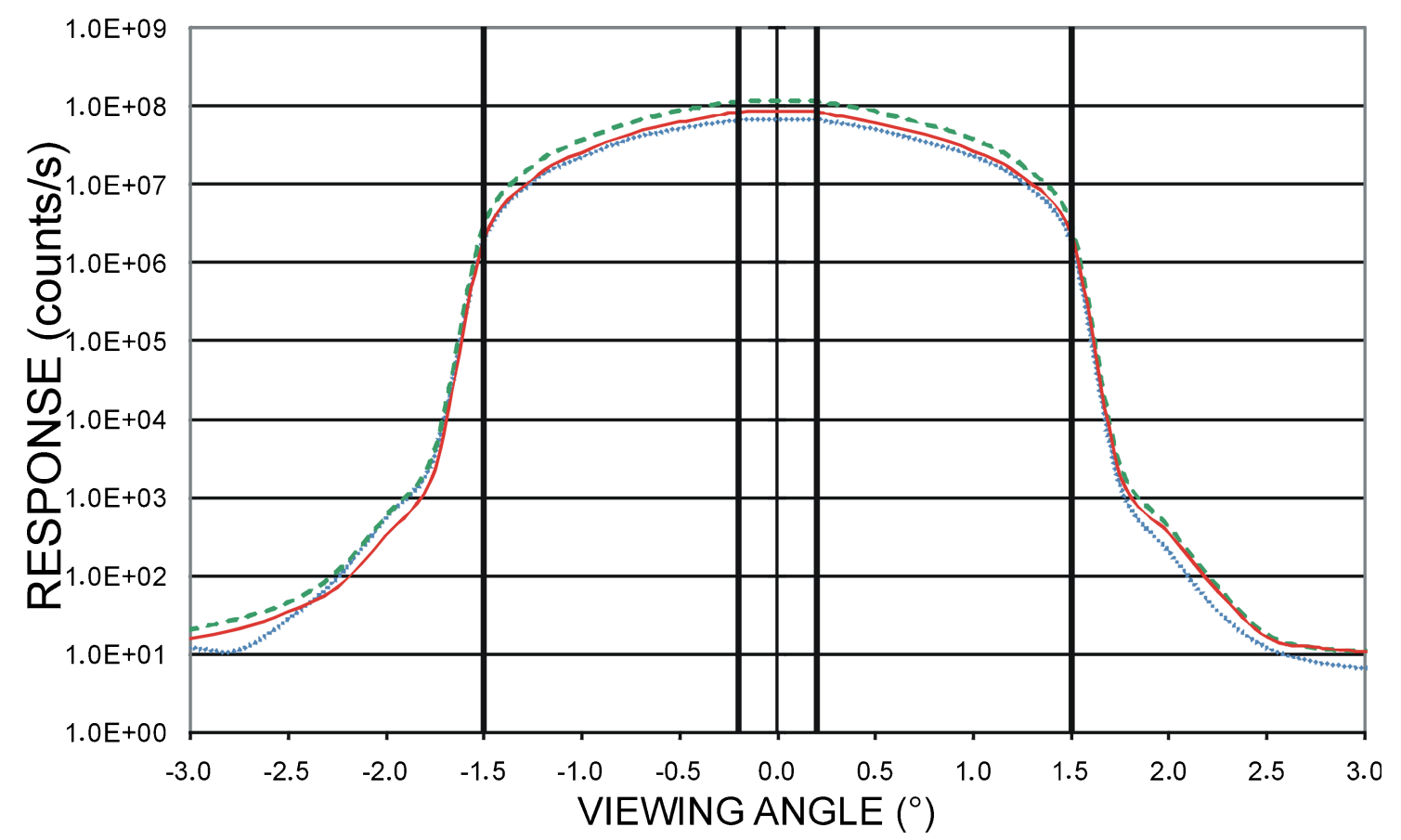

Fig. 5. (Color online) Instrument signature at $400 \mathrm{~nm}$ (dotted curve), $550 \mathrm{~nm}$ (dashed curve), and $700 \mathrm{~nm}$ (full curve).

$2.4 \%$. This is in correspondence with the experimental values.

The second region of the instrument signature comprises the interval from $\pm 0.2^{\circ}$ to $\pm 1.5^{\circ}$ (see Fig. 5). In this interval, the response decreases because the detector aperture gradually misses the incident light. To avoid very large integration times, the neutral density filter with a transmittance of
$1 \%$ or $10 \%$ is now switched into the light beam. The measurement time increases from $0.024 \mathrm{~s}$ toward $0.13 \mathrm{~s}$. Due to the inserted neutral density filter, the measured signal remains in the same order of magnitude (30,000 to 50,000 counts), resulting in a similar uncertainty range of approximately $2 \%$.

At $\pm 1.5^{\circ}$ off-axis, the detector aperture misses the incident beam totally, and the measured flux falls off

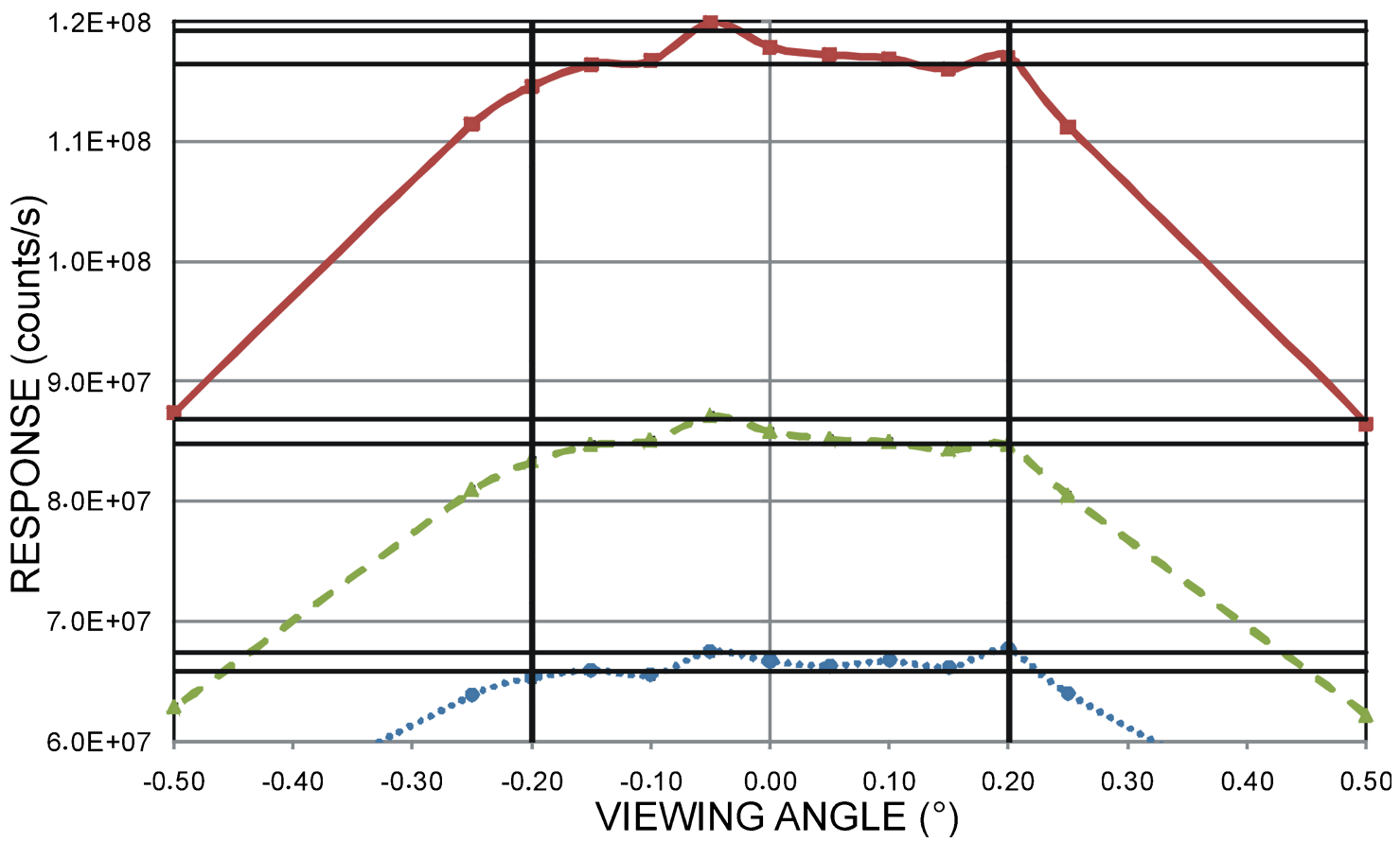

Fig. 6. (Color online) Instrument signature within a small angular interval of $\pm 0.2^{\circ}$ off-axis at three wavelengths: $400 \mathrm{~nm}$ (dotted curve), $550 \mathrm{~nm}$ (dashed curve), and $700 \mathrm{~nm}$ (full curve). 
dramatically. No neutral density filter is inserted now. With an integration time limited to five seconds, a dual beam and dark current corrected signal between 600 and 1000 counts is recorded. This signal originates from the stray light scattered from the instrument components. The uncertainty on the measurement is now in the range 6.3 to $8.2 \%$. If necessary, this uncertainty can be improved by a further increase of the integration time.

From Fig. 5 the instrument provides a dynamic range of seven decades, in accordance with our requirement. In Table 1, the neutral density filters, integration times, and standard deviations are summarized for the different angular intervals.

\section{B. Spectral Bandwith and Stray Light Analysis}

A He-Ne laser emission line was used to evaluate the spectral bandwidth and to analyze the stray light signal of the spectroradiometer. The normalized relative response, also known as the line spread function, is presented in Fig. 7.

A spectral bandwidth calculated as the full width at half-maximum (FWHM) of $3.5 \mathrm{~nm}$ can be deduced. This is in accordance with the theoretical spectral resolution of $4 \mathrm{~nm}$, as mentioned in Subsection 3.B. Further analysis of Fig. 7 shows that the stray light level is less than $0.1 \%$. These spectral characteristics allow accurate determination of the trichromatic values and color coordinates [50,51]. The shoulder on the immediate left of the sharp peak is possibly caused by interreflections of the detector array surface [51]. If necessary, stray light errors can be reduced by use of a stray-light correction matrix, as described by Zong et al. [52].

\section{Absolute Measurement Capability}

To evaluate the absolute measurement capability of the BSDF instrument, the specular reflection from a UV grade fused silica plano-convex lens with known refractive index over the wavelength range 200-2000 nm (SPX043, diameter $50.8 \mathrm{~mm}$, uncoated) was measured at different angles of incidence $\left(5^{\circ}\right.$ to $50^{\circ}$ from normal incidence). The incident beam was checked to be unpolarized. The measurements were compared to the theoretical reflection values for unpolarized incident radiation calculated from the Fresnel equations, and the results are plotted in Fig. 8.

From Fig. 8, a close agreement within $0.5 \%$ between both the measured and theoretical values is

Table 1. Neutral Density Filter, Integration Time, and Standard Deviation as a Function of Viewing Angle

\begin{tabular}{cccc}
\hline $\begin{array}{c}\text { Viewing } \\
\text { Angle }\left(^{\circ}\right)\end{array}$ & $\begin{array}{c}\text { Neutral Density } \\
\text { Filter }(\%)\end{array}$ & $\begin{array}{c}\text { Integration } \\
\text { Time }(s)\end{array}$ & $\begin{array}{c}\text { Standard } \\
\text { Deviation }(\%)\end{array}$ \\
\hline $0^{\circ}$ to $\pm 0.2^{\circ}$ & 0.1 & 0.023 & 2.4 \\
$\pm 0.2^{\circ}$ to & 1 or 10 & 0.024 to 0.13 & 2.4 \\
$\pm 1.5^{\circ}$ & & & \\
Beyond & - & 5 & 8.2 \\
$\pm 1.5^{\circ}$ & & & \\
\hline
\end{tabular}

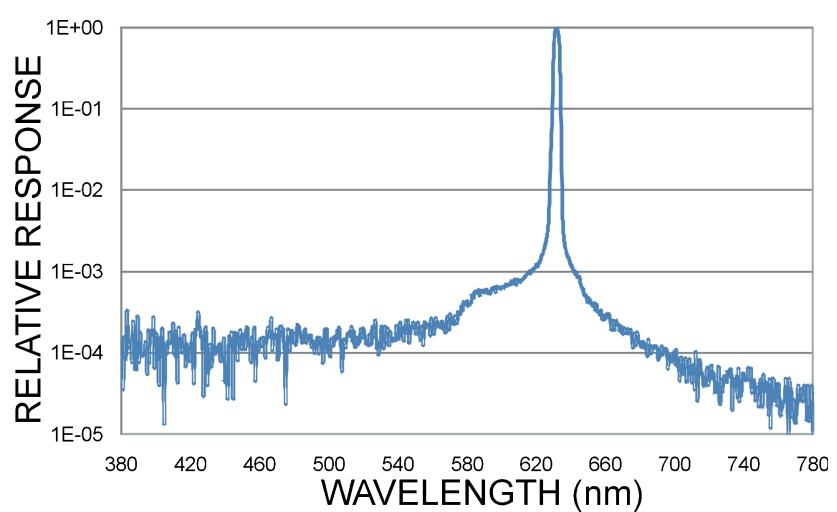

Fig. 7. (Color online) Line spread function of the spectroradiometer.

observed for each incident angle at low wavelengths. Unfortunately, beyond $600 \mathrm{~nm}$, the measured values are lower than expected, with a maximum deviation of $3 \%$.

\section{Measurements}

\section{A. Bidirectional Reflectance Distribution Function of Anisotropic Materials}

Aluminum reflectors and baffles are used for many applications. Anisotropic BRDF characteristics are caused by rolling of the aluminum sheets during production. To clarify the importance of out-of-plane reflectance characterization of such anisotropic samples at oblique angles of incidence, this subsection reports on BRDF measurements on a brushed aluminum reflector (MIRO 7/5000 from Alanod).

Two BRDF characterizations were carried out with an incidence angle of $45^{\circ}$. In the parallel configuration, the incidence plane is chosen parallel to the rolling direction of the sample; in the perpendicular configuration, the incidence plane is chosen perpendicular to the rolling direction. For both configurations, the in-plane (i.e., viewing direction in the incidence plane) and out-of-plane (i.e., viewing direction perpendicular to the incidence plane) BRDF was

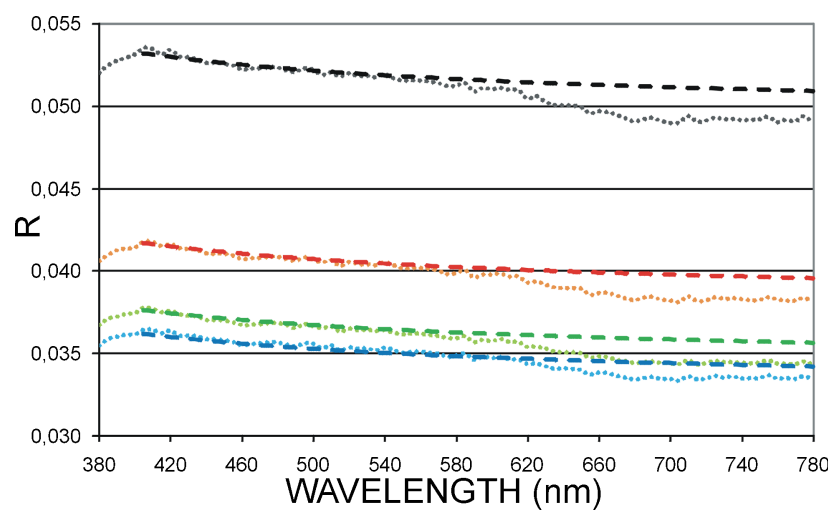

Fig. 8. (Color online) Measurement of the absolute reflection of a UV grade fused silica plano-convex lens (dotted curves) compared to the calculated Fresnel reflection (dashed curves) at incidence angles $10^{\circ}$ (bottom), $30^{\circ}, 40^{\circ}$, and $50^{\circ}$ (top). 
measured. The measurement results for both configurations are presented in Fig. $\underline{9}$ (parallel configuration) and Fig. 10 (perpendicular configuration). The abscissa in Figs. 9 and 10 corresponds to the angular deviation from the specular direction.

From both figures one can observe the influence of the rolling direction on the light distribution. Due to diffraction effects, an important difference in FWHM angle is observed when comparing in-plane to out-ofplane measurements in both configurations. Furthermore, both configurations give rise to different results. These results cannot be obtained by measuring only in the plane of incidence, turning the sample over $90^{\circ}$ around rotation axis $D\left(\delta=90^{\circ}\right)$. This illustrates the importance of a full 3D measurement capability. If such materials are used in luminaires, this anisotropy influences both the light distribution function and the glare when looking into the luminaire. Recent ray tracing software does allow the input of anisotropic BRDFs.

\section{B. Specular Reflection and Regular Transmission of a Dichroic Filter}

Dichroic glass filters are often implemented in luminaires to influence the spectrum of the light source in order to obtain an optimized appearance of meat, fish, or vegetables. Dichroic glass filters are typically produced by vacuum deposition of layers of thin oxide films onto the substrate of borofloat glass. Interference effects are responsible for the selective transmittance and reflectance of certain wavelengths. However, these wavelengths are determined by the optical path length through the coating. For this reason, luminaires that incorporate a dichroic filter suffer from important visual color shifts over the illuminated task area.

In Fig. 11, the regular transmittance and the sum of both the regular transmittance and the specular reflectance at different incident angles of a pink dichroic glass filter are presented. The interference effects are clearly noticeable from the shift in the transmission function toward the shorter wave-

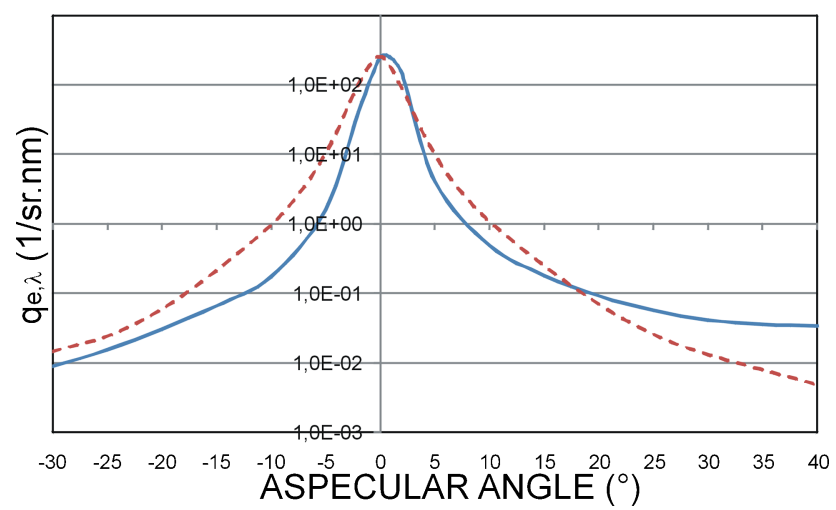

Fig. 9. (Color online) In-plane (full curve) and out-of-plane (dashed curve) BRDF measurement at $550 \mathrm{~nm}$ of a brushed aluminum reflector (MIRO 7/5000 from Alanod) in the parallel configuration (incidence plane parallel to the rolling direction).

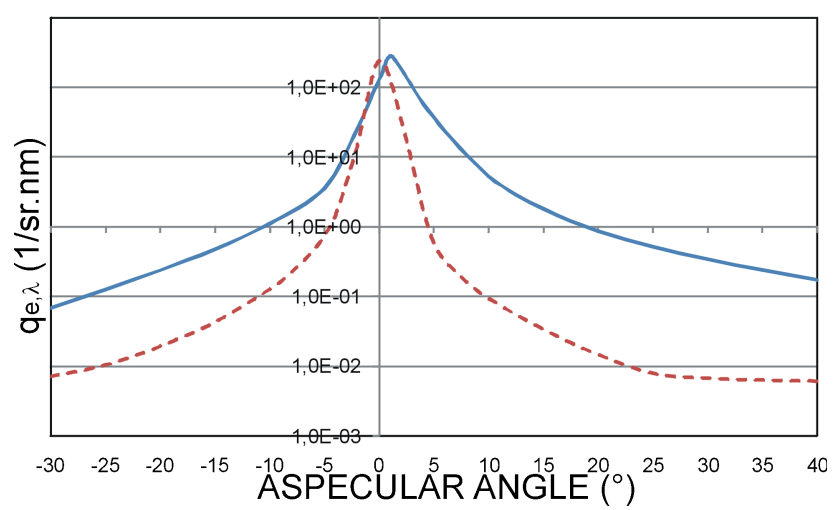

Fig. 10. (Color online) In-plane (full curve) and out-of-plane (dashed curve) BRDF measurement at $550 \mathrm{~nm}$ of a brushed aluminum reflector (MIRO 7/5000 from Alanod) in the perpendicular configuration (incidence plane perpendicular to the rolling direction).

lengths when the incidence angle increases. The perceived color shifts from pink to yellow.

The sum of the regular transmittance and specular reflectance, although individually very wavelength selective, is almost independent of the wavelength and incidence angle. Absorption is limited to 3\%, increasing to $5 \%$ at near UV wavelengths. Due to these highly reflecting coatings, luminaire designers must take into consideration the back reflectance of the filter into the luminaire.

\section{Chromaflair Samples}

ChromaFlair (JDS Uniphase Corporation) is a material that shows dramatic color effects. It consists of small flakes (thickness of the order of $1 \mu \mathrm{m}$ ) that are built up of a highly transparent layer on an opaque reflecting metal and covered by a semitransparent absorber [53]. The color dependency on the viewing angle originates from interference of the light in the transparent layer and from selective absorption in the top layer. Several standard colors are currently available. A typical color name is, for example, "Red/Gold 000." The name reflects the colors seen at approximately $0^{\circ}$ and $45^{\circ}$, respectively. The number represents the approximate hue of the pigment at the normal viewing angle.

For a complete characterization of such objects, one has to measure the color at more than one illumination/viewing angle combination. However, there are no standard geometries and no internationally accepted methods for assessing color variations of such gonio-apparent materials [36]. This has been partly overcome by the introduction of so called multiangle spectrophotometers, which measure at more than one and typically at three to five angle combinations, as proposed by standards DIN 6175-2 [54] and ASTM E2549 [55]. Nevertheless, for many surfaces, notably some of the pearlescent and interference pigments that are now being used for product finishes, the use of more illumination/viewing angles than those proposed in the standard becomes necessary in order to fully characterize the color shift of 


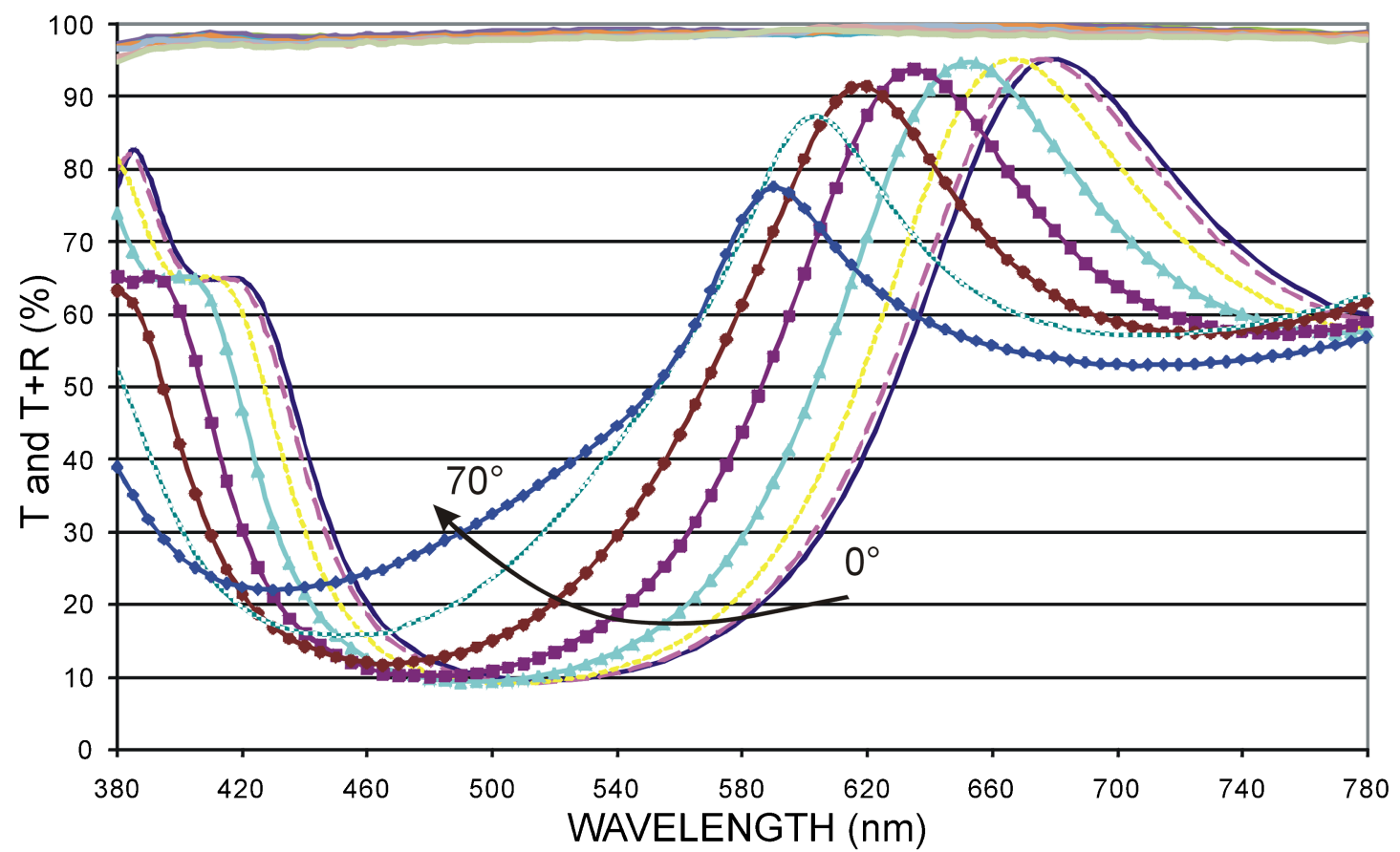

Fig. 11. (Color online) Regular transmittance and sum of both regular transmittance and specular reflectance of a pink dichroic glass filter at incidence angles ranging from $0^{\circ}$ to $70^{\circ}$, with a $10^{\circ}$ increment.

such materials. Nadal [56], for example, suggested a new color measurement instrument with three illumination angles and five viewing angles.

Spectral BRDF measurements can offer interesting insight on how to characterize special-effect colors [57]. As an example, CIELAB colorimetric values, calculated from measurements of the spectral BRDF $q_{e, \lambda}$ on three ChromaFlair samples, are presented in Fig. 12. The colorimetric quantities are calculated for CIE Standard Illuminant D65 and the CIE $10^{\circ}$ Standard Observer. In agreement with the DIN standard, the angle of incidence is taken at $45^{\circ}$ from the surface normal. The viewing angle $\gamma$ is expressed in terms of the "Effektwinkel" or aspecular angle, i.e., the angle referenced to the specular reflection angle (see Fig. 13). $\gamma$ is taken to be positive toward the incident light direction and negative away from it, in agreement with DIN 6175-2. Viewing angles range from $\gamma=-40^{\circ}$ to $\gamma=125^{\circ}$ with a $5^{\circ}$ angle interval in the direction indicated by the arrow. While the DIN standard suggests measuring at angles $\gamma=25^{\circ}, \gamma=45^{\circ}$, $\gamma=75^{\circ}$, and $\gamma=110^{\circ}$, it is clear that these values are covering only a very small region of the potential color shifts (marked by ellipses in Fig. 12). However, the color shift for negative viewing angles is more impressive.

\section{Conclusions}

The spectral BSDF offers a complete description of the spectral and spatial optical characteristics of a material. A measuring instrument allowing for the absolute determination of the spectral BSDF, with a full 3D spatial coverage in both reflectance and transmittance mode, a broadband spectral coverage, a large dynamic range, a reasonable acquisition time, and a large sample illumination area is presented.

The optical layout, angular setup, and related coordinate systems of the instrument have been described in detail. Spectral and spatial characteristics have been determined experimentally. A dynamic range of seven decades and integration times below $5 \mathrm{~s}$ were achieved using neutral density filters. The large sample illumination area combined with the

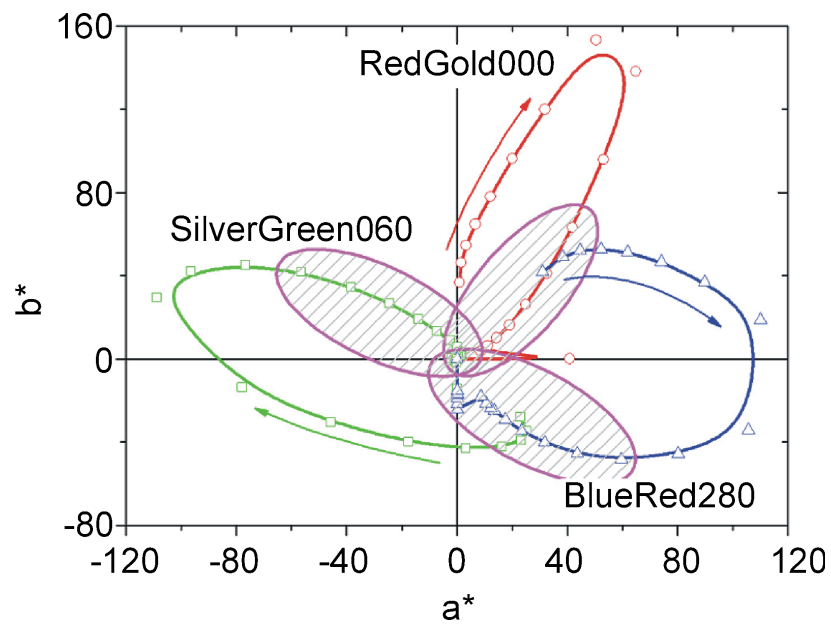

Fig. 12. (Color online) Representation of the CIELAB values in combination with the CIE Standard Illuminant D65 and the CIE $10^{\circ}$ Standard Observer, as calculated from measurements of the spectral BRDF $q_{e, \lambda}$ on three ChromaFlair samples. The angle of incidence was fixed at $45^{\circ}$ from the surface normal. Referring to DIN 6175-2, viewing angles ranged from $\gamma=-40^{\circ}$ to $\gamma=125^{\circ}$ with a $5^{\circ}$ angle interval in the direction indicated by the arrow. 


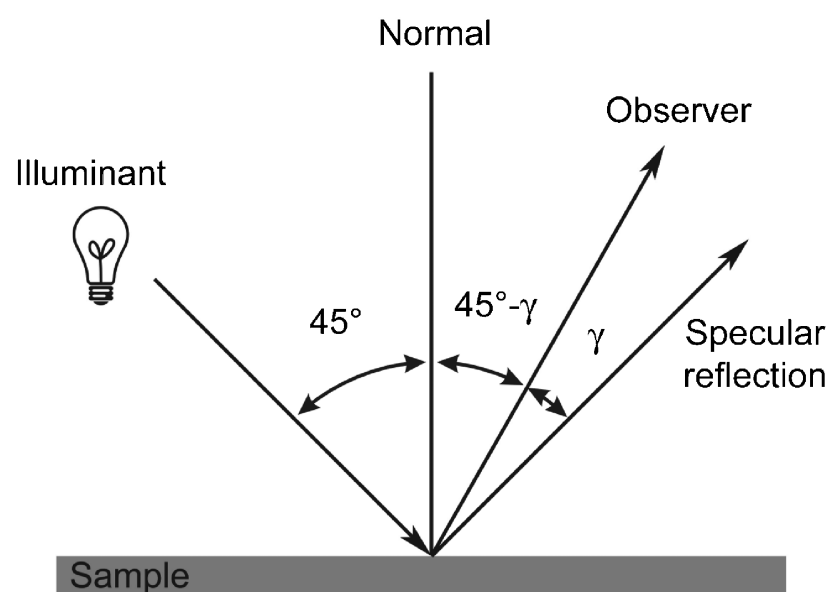

Fig. 13. Measurement geometry according to DIN 6175-2. The angle of incidence is fixed at $45^{\circ}$ from the surface normal. The viewing angle $\gamma$ is expressed in terms of the "Effektwinkel" or aspecular angle, i.e., the angle referred to the specular reflection angle. $\gamma$ is taken to be positive toward the incident light direction.

absolute measurement requirement results in a moderate angular resolution of a few degrees. Measurement uncertainties of approximately $2 \%$ over the visible wavelength range have been obtained. A spectral bandwidth of $3.5 \mathrm{~nm}$ (FWHM) and stray light levels less than $0.1 \%$ allow for an accurate determination of the trichromatic values and color coordinates.

Finally, the capabilities of the instrument were illustrated with some measurements. The necessity for a full 3D measurement capacity was justified by measuring anisotropic aluminum samples. Combined spatial and spectral characteristics in both reflectance and transmittance mode of specular dichroic filters and of diffuse Chromaflair special-effect colors illustrate the versatility of the instrument, providing spectral BSDF data that can be used in a wide range of applications.

\section{Appendix A}

The maximum signal is obtained when measuring the incident flux $\Phi_{\text {in }}$. A typical low signal is obtained when measuring a dark Lambertian reflecting material with a reflectance value of $5 \%$. In this case, the flux reaching the detector $\Phi_{d}$ an be calculated as

$$
\Phi_{d}=L A_{s} \cos \theta_{s} \frac{A_{d}}{d^{2}}
$$

with $L$ and $A_{s}$ the radiance and illuminated area of the sample, respectively, $A_{d}$ the area of the detector head, and $d$ the distance to the sample.

The relation between the radiance and the incident flux is given by

$$
L A_{s}=\frac{0.05 \Phi_{\text {in }}}{\pi}
$$

By substituting Eq. (A2) into Eq. (A1), the ratio of both signals can be written as

$$
\frac{\Phi_{d}}{\Phi_{\text {in }}}=\frac{0.05 \cos \theta_{s} A_{d}}{\pi d^{2}} .
$$

Using the numerical values $\cos \theta_{s}=0.1$, according to a maximum detection angle of about $85^{\circ}$, $d=765 \mathrm{~mm}$, and $A_{d}=\pi r^{2}$ with $r=12.7 \mathrm{~mm}$, a flux ratio of approximately $10^{-6}$ is obtained.

\section{Appendix B}

In Fig. 14, vector $\mathbf{B}_{0}$ is rotated around vector $\mathbf{A}$ over an angle $\theta$. This results in a new vector $\mathbf{B}_{1}$. The positive direction is determined by the right hand rule. Some intermediate vectors are introduced (see Fig. 14).

The projection of $\mathbf{B}_{0}$ and $\mathbf{B}_{1}$ on $\mathbf{A}$ gives rise to vector $\mathbf{C}$, lying parallel or antiparallel with $\mathbf{A}$ :

$$
\mathbf{C}=\left|\mathbf{B}_{0}\right| \cos \alpha \frac{\mathbf{A}}{|\mathbf{A}|},
$$

with $\alpha$ the angle between $\mathbf{A}$ and $\mathbf{B}_{0}$. In the plane perpendicular to $\mathbf{A}, \mathbf{D}_{0}$ and $\mathbf{D}_{1}$ are defined as

$$
\mathbf{D}_{0}=\mathbf{B}_{0}-\mathbf{C}, \quad \mathbf{D}_{1}=\mathbf{B}_{1}-\mathbf{C} .
$$

The second equation defining $\mathbf{B}_{1}$ is given by the projection of $\mathbf{B}_{1}$ on $\mathbf{A}$ :

$$
\mathbf{B}_{1} \cdot \mathbf{A}=\mathbf{B}_{0} \cdot \mathbf{A}=\left|\mathbf{B}_{0} \| \mathbf{A}\right| \cos \alpha .
$$

The projection of $\mathbf{D}_{1}$ on $\mathbf{D}_{0}$ and $\mathbf{D}_{1}$ on $\mathbf{A} \times \mathbf{D}_{0}$ uniquely defines the position of $\mathbf{D}_{1}$ :

$$
\begin{gathered}
\mathbf{D}_{1} \cdot \mathbf{D}_{0}=\left|\mathbf{D}_{1}\right|\left|\mathbf{D}_{0}\right| \cos \theta, \\
\mathbf{D}_{1} \cdot\left(\mathbf{A} \times \mathbf{D}_{0}\right)=\left|\mathbf{D}_{1}\right|\left|\mathbf{A} \times \mathbf{D}_{0}\right| \sin \theta .
\end{gathered}
$$

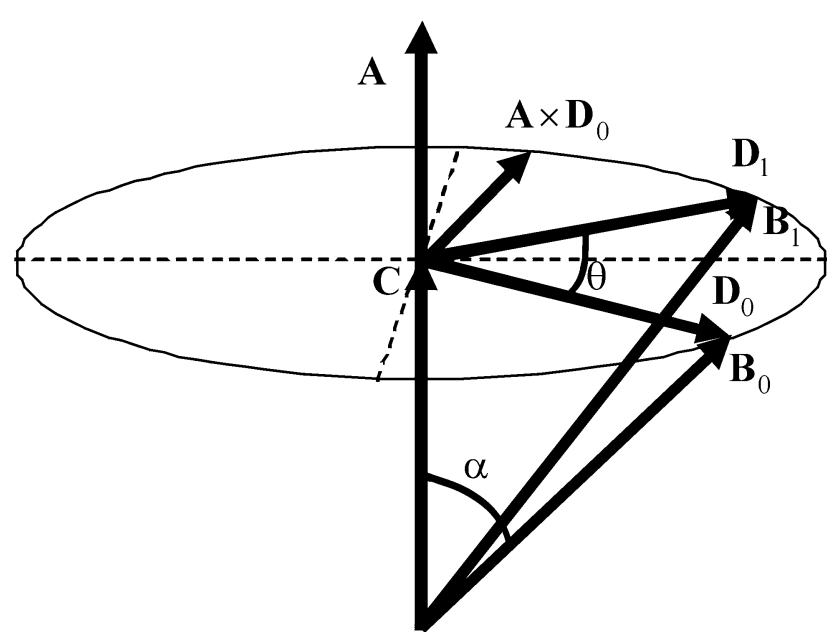

Fig. 14. Rotation of vector $\mathbf{B}_{0}$ over a positive angle $\theta$ around vector $\mathbf{A}$. 
Equations (B4) and (B5) must be reformulated in terms of $\mathbf{B}_{0}$ and $\mathbf{B}_{1}$ making use of Eqs. (B1) and (B2). Equation (B4) can be rewritten as

$$
\mathbf{B}_{1} \cdot \mathbf{B}_{0}-\left|\mathbf{B}_{0}\right|^{2} \cos ^{2} \alpha=\left|\mathbf{B}_{0}\right|^{2} \sin ^{2} \alpha \cos \theta
$$

or

$$
\mathbf{B}_{1} \cdot \mathbf{B}_{0}=\left|\mathbf{B}_{0}\right|^{2}\left(\cos \theta+\frac{\left(\mathbf{B}_{0} \cdot \mathbf{A}\right)^{2}}{\left|\mathbf{B}_{0}\right|^{2}|\mathbf{A}|^{2}}(1-\cos \theta)\right) .
$$

For Eq. (B5) we can find

$$
\begin{aligned}
\mathbf{B}_{1} \cdot\left(\mathbf{A} \times \mathbf{B}_{0}\right) & =\left|\mathbf{D}_{0}\|\mathbf{A}\| \mathbf{D}_{0}\right| \sin \theta \\
& =|\mathbf{A}|\left|\mathbf{B}_{0}\right|^{2}\left[1-\cos ^{2} \alpha\right] \sin \theta
\end{aligned}
$$

or

$$
\mathbf{B}_{1} \cdot\left(\mathbf{A} \times \mathbf{B}_{0}\right)=|\mathbf{A}|\left|\mathbf{B}_{0}\right|^{2}\left[1-\frac{\left(\mathbf{B}_{0} \cdot \mathbf{A}\right)^{2}}{\left|\mathbf{B}_{0}\right|^{2}|\mathbf{A}|^{2}}\right] \sin \theta .
$$

Equations (B3), (B7), and (B9) are three linear equations that can be numerically solved for the three components of $\mathbf{B}_{1}$. The results can be written in matrix notation as

$$
\left(\begin{array}{c}
B_{1 x} \\
B_{1 y} \\
B_{1 z}
\end{array}\right)=\mathbf{R}(\mathbf{A}, \theta) \cdot\left(\begin{array}{c}
B_{0 x} \\
B_{0 y} \\
B_{0 z}
\end{array}\right)
$$

with $\mathbf{R}(\mathbf{A}, \theta)$ the rotation matrix around $\mathbf{A}$ over an angle $\theta$ explicitly given by

$$
\mathbf{R}(\mathbf{A}, \theta)=\frac{1}{|\mathbf{A}|}\left(\begin{array}{ccc}
A_{x}^{2}+\cos \theta\left(A_{y}^{2}+A_{z}^{2}\right) & A_{x} A_{y}(1-\cos \theta)-A_{z}|\mathbf{A}|^{1 / 2} \sin \theta & A_{x} A_{z}(1-\cos \theta)+A_{y}|\mathbf{A}|^{1 / 2} \sin \theta \\
A_{x} A_{y}(1-\cos \theta)+A_{z}|\mathbf{A}|^{1 / 2} \sin \theta & A_{y}^{2}+\cos \theta\left(A_{x}^{2}+A_{z}^{2}\right) & A_{y} A_{z}(1-\cos \theta)-A_{x}|\mathbf{A}|^{1 / 2} \sin \theta \\
A_{x} A_{z}(1-\cos \theta)-A_{y}|\mathbf{A}|^{1 / 2} \sin \theta & A_{y} A_{z}(1-\cos \theta)+A_{x}|\mathbf{A}|^{1 / 2} \sin \theta & A_{z}^{2}+\cos \theta\left(A_{x}^{2}+A_{y}^{2}\right)
\end{array}\right)
$$

\section{References}

1. J. C. Stover, Optical Scattering: Measurement and Analysis, 2nd ed. (SPIE, 1995).

2. G. J. Ward, "Measuring and modeling anisotropic reflection," Comput. Graph. 26, 265-272 (1992).

3. K. F. Karner, H. Mayer, and M. Gervautz, "An image based measurement system for anisotropic reflection," in Proceedings of the Eurographics Workshop on Rendering Techniques '96 (Springer-Verlag, 1996), Vol.15(3), pp.119-128.

4. S. C. Foo, "A gonioreflectometer for measuring the bidirectional reflectance of material for use in illumination computation," Master's thesis (Cornell University, 1997).

5. B. van Ginneken, M. Stavridi, and J. J. Koenderink, "Diffuse and specular reflectance from rough surfaces," Appl. Opt. 37, 130-139 (1998).

6. K. J. Dana, S. Nayar, B. van Ginneken, and J. J. Koenderink, "Reflectance and texture of real-world surfaces," ACM Trans. Graph. 18, 1-34 (1999).

7. S Marschner, S. H. Westin, E. P. F. Lafortune, and K. E. Torrance, "Image-based bidirectional reflectance distribution function measurement," Appl. Opt. 39, 2592-2600 (2000).

8. D. K. McAllister, "A generalized surface appearance representation for computer graphics," Ph.D dissertation (University of North Carolina, 2002).

9. J. A. Paterson, D. Claus, and A. W. Fitzgibbon, "BRDF and geometry capture from extended inhomogeneous samples using flash photography," in Proceedings of the Eurographics Workshop on Rendering Techniques '05 (Springer-Verlag, 2005), Vol. 24(3), pp.383-391.

10. K. Vom Berge, "Compact factored representation for reflectance of complex materials,” Ph.D. dissertation (K.U. Leuven, 2006).
11. H. Li, S.-C. Foo, K. E. Torrance, and S. H. Westin, "Automated three-axis gonioreflectometer for computer graphics applications", Opt. Eng. 45, 043605 (2006).

12. A. Ghosh, S. Achutha, W. Heidrich, and M. O'Toole, "BRDF acquisition with basis illumination," in Proceedings of IEEE International Conference on Computer Vision (IEEE, 2007).

13. W. C. Snyder, "Reciprocity of the bidirectional reflectance distribution function (BRDF) in measurements and models of structured surfaces," IEEE Trans. Geosci. Remote Sens. 36, 685-691 (1998).

14. Y. Boucher, H. Cosnefroy, D. Petit, G. Serrot, and X. Briottet, "Comparison of measured and modeled BRDF of natural targets," Proc. SPIE 3699, 16-26 (1999).

15. S. R. Sandmeier and K. I. Itten, "A field goniometer system (FIGOS) for acquisition of hyperspectral BRDF data," IEEE Trans. Geosci. Remote Sens. 37, 978-986 (1999).

16. J. R. Shell, Bidirectional Reflectance: An Overview with Remote Sensing Applications \& Measurement Recommendations (Rochester Institute of Technology, 2004).

17. D. Combes, L. Bousquet, S. Jacquemoud, H. Sinoquet, C. Varlet-Grancher, and I. Moya, "A new spectrogoniophotometer to measure leaf spectral and directional optical properties," Remote Sens. Environ. 109, 107-117 (2007).

18. P. Apian-Bennewitz and J. von der Hardt, "Enhancing and calibrating a goniophotometer," Solar Energy Mater. Sol. Cells 54, 309-322 (1998).

19. M. Andersen, "Validation of the performance of a new bidirectional video-goniophotometer," Light. Res. Technol. 38, 295313 (2006).

20. J.-M. Coulomb and C. Brusque, "Propriétés de réflexion des surfaces. Le gonioréflectomètre du LCPC. Performances et 
perspectives d'études," Bull. Lab. Ponts Chaussées 204, 2535 (1996).

21. H. Granberg, "Optical response from paper. Angle-dependent light scattering: measurements, modelling, and analysis," Ph.D. dissertation (KTH Royal Institute of Technology, 2003).

22. J. Faucheu, K. A. Wood, L.-P. Sung, and J. W. Martin, "Relating gloss loss to topographical features of a PVDF coating," J. Coat. Technol. 3, 29-39 (2006).

23. M.-C. Béland and L. Mattsson, "Optical print quality of coated papers,” J. Pulp Pap. Sci. 23, 493-498 (1997).

24. J. S. Arney, H. Heo, and P. G. Anderson, "A micro-goniophotometer and the measurement of print gloss,"J. Imag. Sci. Technol. 48, 458-463 (2004).

25. M. Lindstrand, "An angularly and spatially resolved reflectometer for a perceptually adequate characterization of gloss," J. Imag. Sci. Technol. 49, 71-84 (2005).

26. D. H. Goldstein and D. B. Chenault, "Spectropolarimetric reflectometer," Opt. Eng. 41 (5), 1013-1020 (2002).

27. J. F. Murray-Coleman and A. M. Smith, "The automated measurement of BRDFs and their application to luminaire modelling," J. Illum. Eng. Soc. (1990).

28. A. A. Letunov, B. H. Barladian, E. Y. Zueva, V. P. Veshnevetc, and S. A. Soldatov, "CCD-based device for BRDF measurements in computer graphics," presented at GraphiCon 99The 9th International Conference on Computer Graphics and Vision, Moscow, Russia, 26 August-1 September, 1999.

29. F. Drago and K. Myszkwski, "Validation proposal for global illumination and rendering techniques," Comput. Graph. 25, 511-518 (2001).

30. J.-M. Deniel, "Modélisation des luminaires et des BRDF : réalisation, mesure et compression," $\mathrm{Ph} . \mathrm{D}$ dissertation (Université de Rennes 1, 2002).

31. Commission Internationale de l'Eclairage Technical Report 130:1998, "Practical methods for the measurement of reflectance and transmittance" (CIE, 1998).

32. Commission Internationale de l'Eclairage Technical Report 15:2004, "Colorimetry" (CIE, 2004).

33. Commission Internationale de l'Eclairage Technical Report 176:2006, "Geometric tolerances for colour measurements" (CIE, 2006).

34. American Society for Testing and Materials Standard E179, "Standard guide for selection of geometric conditions for measurement of reflection and transmission properties of materials" (ASTM, 1996).

35. American Society for Testing and Materials Test Method D523, "Test method for specular gloss" (ASTM, 1999).

36. A. Gilchrist, "Characterizing special-effect colours," Surf. Coat. Intl. B 85, 281-285 (2002).

37. F. E. Nicodemus, J. C. Richmond, and J. J. Hsia, "Geometrical considerations and nomenclature for reflectance," Natl. Bur. Stand. Monogr. 160, (1977).

38. D. R. White, P. Saunders, S. J. Bonsey, J. van de Ven, and H. Edgar, "Reflectometer for measuring the bidirectional reflectance of rough surfaces," Appl. Opt. 37, 3450-3454 (1998).
39. T. A. Germer and C. C. Asmail, "Goniometric optical scatter instrument for out-of-plane ellipsometry measurements," Rev. Sci. Instrum. 70, 3688-3695 (1999).

40. G. Obein, R. Bousquet, and M. E. Nadal, "New NIST reference goniospectrometer," Proc. SPIE 5880, 241-250 (2005).

41. W. Erb, "Computer-controlled gonioreflectometer for the measurement of spectral reflection characteristics," Appl. Opt. 19, 3789-3794 (1980).

42. X. Feng, J. R. Schott, and T. Gallagher, "Comparison of methods for generation of absolute reflectance-factor values for bidirectional reflectance-distribution functions studies," Appl. Opt. 32, 1234-1242 (1993).

43. M. R. Pointer, N. J. Barnes, P. J. Clarke, and M. J. Shaw, "A new goniospectrophotometer for measuring gonio-apparent materials," Color. Technol. 121, 96-103 (2005).

44. K. J. Dana, "CUReT: Columbia Utrecht reflectance and texture database," http://www1.cs.columbia.edu/CAVE/software/ curet/index.php.

45. G. Obein, T. Leroux, K. Knoblauch, and F. Viénot, "Visually relevant gloss parameters," presented at the 11th International Metrology Congress, Toulon, France, 20-24 October 2003.

46. R. Lu, J. J. Koenderink, and A. M. L. Kappers, "Optical properties (bidirectional reflection distribution functions) of velvet," Appl. Opt. 37, 5974-5984 (1998).

47. H. P. A. Lensch, J. Kautz, and M. Goesele, "Image-based reconstruction of spatial appearance and geometric Detail," ACM Trans. Graph. 22, 234 (2003).

48. W. Matusik, H. Pfister, M. Brand, and L. McMillan, "A datadriven reflectance model," ACM Trans. Graph. 22, 759-769 (2003).

49. American Society for Testing and Materials Standard E1392, "Standard practice for angle resolved optical scatter measurements on specular or diffuse surfaces" (ASTM, 1996).

50. H. J. Kostkowski, "Reliable spectroradiometry" (Spectroradiometry Consulting, 1997).

51. Y. Ohno, "Spectral colour measurement," in Colorimetry, Understanding the CIE System, 1st ed., J. Schanda, ed. (CIE Central Bureau, 2006).

52. Y. Zong, S. W. Brown, K. R. Lykke, and Y. Ohno, "Stray light correction method for array spectrometers," Appl. Opt. 45, 1111-1119 (2006).

53. "Chromaflair: thin-film interference color," Technical Note (JDS Uniphase Corporation, 2006).

54. Deutsches Institut für Normung Standard 6175-2, "Colour tolerances for automobile lacquer finishes, Part 2: Effect lacquer finishes" (DIN, 2001).

55. American Society for Testing and Materials Standard E254908, "Standard practice for multi-angle measurement of interference pigments" (ASTM, 2008).

56. M. E. Nadal and T. A. Germer, "Colorimetric characterization of pearlescent coatings," Proc. SPIE 4421, 757-760 (2002).

57. F. Leloup, P. Hanselaer, M. Pointer, and J. Versluys, "Characterization of gonio-apparent colours," in Proceedings of the 10th Congress of the International Color Association, J. L. Nieves and J. Hernández-Andrés, eds. (CIE, 2005), pp. 515-518. 